\title{
Composición, estructura y diversidad de poblaciones de Nothofagus glauca ubicadas en la zona mediterránea de Chile
}

\section{Composition, structure and diversity of populations of Nothofagus glauca located in the Mediterranean zone of Chile}

\author{
Fernando Muñoz ${ }^{1 *}$, Carolina Muñoz ${ }^{1}$, Matilde Uribe ${ }^{1}$, María Ángela Martín², Juan Ramón Molina ${ }^{2}$, \\ Miguel Ángel Herrera², Juan Bautista Álvarez² \& Luís Miguel Martín² \\ 'Universidad de Concepción, Facultad de Ciencias Forestales, Victoria 631, Concepción, Chile. \\ 2Universidad de Córdoba, Escuela Técnica Superior de Ingenieros Agrónomos y de Montes, Córdoba, España. \\ *fmunoz@udec.cl
}

\begin{abstract}
RESUMEN
Los bosques de Nothofagus glauca se encuentran ubicados en la zona mediterránea de Chile central. Su eliminación para habilitar suelo para uso agrícola, forestal y ganadero, más la utilización de su madera para leña y carbón, redujo drásticamente su superficie. Se estudiaron un total de cuatro bosques de $N$. glauca localizados dos en la Cordillera de la Costa y dos en la Cordillera de los Andes de Chile central. A través de inventario forestal, durante el verano de los años 2008 y 2009 , se determinó la composición, la estructura y la diversidad de las comunidades habitadas por $N$. glauca, y se cosechó semillas escalando árboles seleccionados con el propósito de obtener información de la diversidad genética de la especie. Los resultados indicaron que existe gran diferencia entre los bosques de $N$. glauca ubicados en la costa y cordillera de los Andes. Los bosques costeros presentaron mayor riqueza dentro del rodal; dominancia y diversidad de especies entre rodales. En los bosques estudiados, N. glauca fue la especie principal en todos los estratos de copa (dominante, codominante y suprimido), con estructuras diamétricas principalmente del tipo "J invertida". La mejor semillación, en cantidad y calidad, ocurrió en los bosques costeros.
\end{abstract}

Palabras clave: Nothofagus, comunidades vegetales, diversidad alfa y beta, riqueza de especies.

\begin{abstract}
The forests of Nothofagus glauca are located in the mediterranean zone of central Chile. Its elimination to enable soil for agricultural, forestry, more cattle use as well as the utilization of its wood for fuelwood and coal, reduced drastically its surface. Forests of N. glauca, that are located in the Coast and Andes chain mountain of the central zone of Chile, were studied. Across forest inventory, during summer of 2008 and 2009, it was recorded the composition, structure and diversity of the communities inhabited by $N$. glauca, some of which were selected to be climbed for seed collection to be used for genetic diversity studies. The results indicated a great difference between the forests of $N$. glauca located in Coast and the Andes. The forests of the Coast chain mountain, presented major species richness of each stand, dominance and diversity of species between communities. In all studied forests, $N$. glauca was the predominant species in all strata of crown (dominant, codominant, suppressed), with diameter class structures principally of the type "inverse J-shaped". The best seed production, in quantity and quality, was registered in Coast chain mountain forest.
\end{abstract}

KEYwords: Nothofagus, plant communities, alpha and beta diversity, species richness.

\section{INTRODUCCIÓN}

Los bosques de Nothofagus glauca (Phil.) Krasser, ubicados en la zona mediterránea de Chile central, han sufrido grandes disturbios debido a su eliminación para habilitar suelo para uso agrícola, forestal y ganadero, y al uso de su madera para leña y carbón (Serra et al. 1986).
A mediados de la década del 70 la superficie de estos bosques era alrededor de 900.000 hectáreas (Urzúa 1975), disminuyendo a sólo 188.323 ha (tipo forestal roblehualo) (CONAF-CONAMA-BIRF 1999). Esto llevó a clasificar a la especie en la categoría "vulnerable" según el Libro Rojo de la Flora Terrestre de Chile (Benoit 1989) y por Hechenleitner et al. (2005), en la actualidad tiene la 
categoría de "Casi Amenazada" (CONAMA 2012), aunque la Unión Internacional para la Conservación de la Naturaleza mantiene la categoría de vulnerable (IUCN, 2012),

Nothofagus glauca es un árbol endémico de Chile central considerado como la especie más representativa de los bosques mediterráneos del género Nothofagus (Donoso 1993). Es conocido comúnmente como hualo, roble, roble colorado o roble maulino. Especie común en los tipos forestales roble-hualo y ciprés de la cordillera (Del Fierro $\&$ Pancel 1998). Su mayor extensión se encuentra en forma de renovales, de estructura, composición y densidad muy heterogéneas (Serra et al. 1986).

Los bosques de $N$. glauca presentan distribución discontinua entre la Cordillera de la Costa y de Los Andes desde la provincia del Cachapoal en la Región del Libertador Bernardo O’Higgins hasta la provincia del Biobío en la Región del Biobío (Hechenleitner et al. 2005). El límite sur se encuentra ubicado a $0,5 \mathrm{~km}$ al noreste del poblado de Quilleco, en el límite oriente del Llano Central en la provincia del Biobío (Le-Quesne \& Sandoval 2001). En la Cordillera de la Costa se distribuye principalmente desde la localidad de Alhué, al sur de la Región Metropolitana hasta el río Itata, Región del Bíobío, entre los 150 y 800 m s.n.m. En la Cordillera de los Andes se presenta en grandes masas boscosas, desde la precordillera de Molina en la Región del Maule hasta la localidad de San Fabián de Alico en la Región del Biobío, bajo los 1.200 m s.n.m. La mayor superficie de esta especie se localiza en la costa de las provincias de Talca y Cauquenes (Región del Maule). Se encuentra protegida en las reservas nacionales Los Ruiles y Los Queules (Cordillera de la Costa) y Radal 7 Tazas y Altos de Lircay (Cordillera de los Andes) (Hechenleitner et al. 2005).

La distribución discreta de las poblaciones dispone a la especie de unas características particularmente susceptibles ante la deforestación y degradación del bosque. Por lo tanto, las poblaciones existentes se encuentran sometidas a un intenso proceso de fragmentación. Su asociación con especies relevantes en conservación como Nothofagus alessandrii Esp., Beilschmiedia berteroana Kosterm. y Nothofagus leonii Espinosa, le confieren indudable valor ecológico y paisajístico, tanto por si mismo como por las relaciones en su hábitat nativo. Se considera a $N$. glauca como una especie clave dentro de determinados hábitats y su pérdida o continua disminución puede impactar directamente sobre otras especies, debido a que dentro de los bosques de esta especie se produce la mayor cantidad de endemismo y ocurrencia de especies leñosas raras y amenazadas de extinción en Chile, entre las cuales destacan Gomortega keule (Molina) Baill., Beilschmiedia berteroana (Gay) Kosterm., Pitavia punctata (Ruiz \& Pav.) Molina, Eucryphia glandulosa (Reiche) (actualmente Eucryphia glutinosa (Poepp. \& Endl.) Baill.) y Legrandia concinna (Phil.) Kausel (Donoso 1993).

Esta discontinuidad espacial determina una amplia diversidad de asociaciones con especies nativas. Las zonas más húmedas caracterizan un bosque mixto junto a Nothofagus obliqua (Mirb.) Oerst. $y$ el híbrido $N$. leonii. Los fondos de los valles andinos pueden presentar un bosque ocasional junto a Nothofagus dombeyi (Mirb.) Oerst. y Nothofagus alpina (Poepp. \& Endl.) Oerst. Las zonas más secas suelen presentar rodales puros de hualo ocasionalmente acompañado de especies endémicas de gran valor paisajístico: Azara petiolaris (D. Don) I.M.Johnst., Gaultheria phillyreifolia (Pers.) Sleumer, Sophora cassioides (Phil.) Sparre.

Biodiversidad es un término abstracto y de difícil definición, que necesita especificarse en cada contexto individual (Del Río et al. 2003). Dentro de este concepto se debe englobar toda clase de variedad natural, desde la diversidad de comunidades a nivel de paisaje (diversidad $\gamma$ ), diferencias entre comunidades (diversidad $\beta$ ) y diversidad de especies dentro de una comunidad (diversidad $\alpha$ ) (Del Río et al. 2003) hasta diversidad genética. Magurran (1989) argumenta tres razones para señalar la importancia de la diversidad biológica: (a) es el eje central de la ecología, (b) las medidas de la biodiversidad aparecen como indicadores del funcionamiento de los ecosistemas y (c) en la actualidad hay gran debate sobre cómo medir la biodiversidad o diversidad biológica. Por otra parte, la determinación de la biodiversidad es un elemento básico para un manejo forestal sustentable.

La especie $N$. glauca presenta serio peligro de conservación. Se encuentra escasamente representada en el Sistema Nacional de Áreas Silvestres Protegidas del Estado (SNASPE), las poblaciones remanentes, especialmente las localizadas en la costa, se encuentran fragmentadas y rodeadas por plantaciones de Pinus radiata D. Don, y sometidas a los procesos antropogénicos que la han llevado a su actual estado de degradación. Adicionalmente, no hay contacto entre las poblaciones costeras y andinas. Urge desarrollar estrategias que permitan la conservación de la especie. Para ello, es necesario conocer las poblaciones remanentes, desde las cuales sea posible obtener material vegetal de calidad que permita la futura restauración ecológica de la especie. Por esta razón, este estudio tiene como objetivo determinar la composición, estructura y diversidad de comunidades de $N$. glauca ubicadas en la Cordillera de la Costa y en la Cordillera de los Andes.

\section{MÉTODOS}

ÁREA DE ESTUDIO

La selección del área de estudio se realizó considerando cobertura vegetal de $N$. glauca, material cartográfico de empresas forestales y fotografías aéreas recientes. Luego de un reconocimiento detallado en terreno se seleccionaron cuatro áreas de estudio, dos áreas ubicadas en la comuna de Pelluhue, provincia de Cauquenes, Región del Maule (Cordillera de la Costa) y otras dos en la comuna de San Fabián de Alico, provincia de Ñuble, Región del Biobío (Cordillera de los Andes). Las áreas ubicadas en la Cordillera de la Costa correspondieron a la reserva nacional Los Queules (35 58' 55, 1' S; 72 41' 13,8” O) y predio Copiulemu ( $36^{\circ} 00^{\prime} 08,4^{\prime \prime} \mathrm{S} ; 7^{\circ} 40^{\prime} 27,9^{\prime \prime} \mathrm{O}$ ) de propiedad de Forestal Celco S.A. Las áreas ubicadas en la Cordillera de los Andes fueron Laguna de La Plata (36 $36^{\circ}$ ' 20,8" S; $\left.71^{\circ} 27^{\prime} 45,9^{\prime \prime} \mathrm{O}\right)$ de propiedad particular y Cerro Castro $\left(36^{\circ}\right.$ $28^{\prime} 40,8^{\prime \prime} \mathrm{S} ; 7^{\circ} 34^{\prime}$ 00,4" O) de propiedad de Forestal Celco S.A. Los sectores seleccionados son representativos de la 
especie, tienen fácil acceso y no se encuentran intervenidos, aunque los bosques costeros se encuentran rodeados de bosques cultivados (plantaciones).

Clima y suelo del ÁRea de estudio

El sector costero, según Novoa \& Villaseca (1989), posee clima mediterráneo con acción moderadora del mar, lo que confiere al sector un clima cálido con variaciones, debido al aumento de la latitud y la altitud (López 1994). El sector tiene inviernos suaves, con estación seca de más de siete meses, temperatura media anual de $15,2{ }^{\circ} \mathrm{C}$ con máxima en el mes más cálido (enero) de $31,3^{\circ} \mathrm{C}$ y mínima en el mes más frío (julio) de $4,6^{\circ} \mathrm{C}$, la humedad relativa es aproximadamente $80 \%$. El régimen hídrico se caracteriza por una precipitación anual de $641,7 \mathrm{~mm}$, siendo el mes de junio el más lluvioso. Los suelos del sector costero provienen preferentemente de granodioritas (Pimstein 1974). Son suelos profundos, en la posición alta y plana de la Cordillera de la Costa. Poseen textura franca de color pardo oscuro en la superficie y textura franco-limosa. Su topografía es de lomajes suaves moderados y físicamente presenta características similares a los suelos "trumaos", especialmente al estado seco. Son suelos muy sueltos en seco, permeables, de buen drenaje y estructuras muy favorables para el desarrollo de las raíces y no presenta erosión hídrica, sin embargo, en verano están sometidos a erosión eólica cuando han tenido cultivos anuales.

La cordillera andina, según Novoa \& Villaseca (1989), cuenta con un clima mediterráneo temperado de veranos muy calurosos y secos (cinco meses). El mes más frío (julio) presenta temperatura mínima media de $3,5^{\circ} \mathrm{C}$ con una máxima media del mes más cálido (enero) de $28,8^{\circ} \mathrm{C}$, la temperatura media anual es de $14^{\circ} \mathrm{C}$. El régimen hídrico se caracteriza por una precipitación media anual de 1.025 $\mathrm{mm}$, siendo el mes de julio el más lluvioso, sin embargo, las lluvias se presentan intensamente durante todo el año. Las heladas son frecuentes e intensas durante el periodo de abril a noviembre. Los suelos en el sector andino están constituidos por rocas graníticas cubiertas de conglomerados volcánicos que originan los suelos conocidos como trumaos (Donoso 2008). Son perfiles profundos, de color pardo grisáceo en húmedo, textura franco-arenosa muy fina, ligeramente plásticos y adhesivos en húmedo, estructura granular fina. A medida que cambia la profundidad, el color cambia a pardo amarillento, sin estructura, con restos de tobas volcánicas. Posee una topografía de lomajes ondulados con pendientes diversas, disectados por numerosos ríos, esteros y quebradas con cursos de agua intermitentes que fluyen hacia la Depresión Central. Estos suelos presentan erosión de manto de intensidad variable, que ha influido notablemente en la disminución de su fertilidad.

INVENTARIO FORESTAL

En cada bosque estudiado (cuatro en total) se realizó inventario forestal del tipo muestreo simple con distribución aleatoria de las muestras (Tabla I). El número de muestras fue de tres por bosque y de tamaño $800 \mathrm{~m}^{2}$.

En cada parcela se registró la especie, DAP (diámetro altura de pecho) de todos los árboles mayores a $5 \mathrm{~cm}$, altura total, dominancia (de acuerdo a la clasificación de Kraft (Daniel et al. 1982)) y calidad fustal (fuste recto, sinuoso, deforme). Adicionalmente, en una muestra representativa de la distribución diamétrica de los árboles se realizó determinación de edad en 19 árboles utilizando taladro de incremento. El trabajo de campo se realizó durante el verano de los años 2008 y 2009, que incluyó recolección de semillas mediante escalamiento de 12 árboles seleccionados en cada bosque (total 48 árboles) según su representatividad en la distribución diamétrica de bosque. Debido a la baja presencia de semillas, especialmente en los bosques andinos, el escalamiento de cada árbol fue necesario repetirlo durante los meses de enero, febrero y marzo de los años 2008 y 2009.

\section{ANÁLISIS DE DATOS}

La composición florística se determinó por medio de la riqueza de especies arbóreas, para lo cual se identificó y contabilizó el número total de especies arbóreas presentes en cada bosque. Para los análisis de estructura de rodal, se construyeron tablas de rodal por bosque. Para los análisis de diversidad, se obtuvo en cada bosque los indicadores de riqueza dentro de una comunidad (diversidad $\alpha$ ) y diversidad entre comunidades (diversidad $\beta$ ). Los indicadores para diversidad $\alpha$ utilizados fueron: [1] Índice de Simpson y [2] Índice de Shannon (Del Río et al. 2003, Pauchard et al. 2006). Los indicadores de diversidad $\beta$ utilizados son los índices de similitud [3] Índice de Jaccard e [4] Índice de Sorensen. Los indicadores mencionados se determinaron mediante las siguientes expresiones:

$$
\begin{aligned}
& \text { Índice de Simpson : } 1-D=1-\sum p_{i}^{2} \\
& \text { Índice de Shannon : } H^{\prime}=-\sum p_{i} \ln p_{i} \\
& \text { Índice de Jaccard }=\frac{c}{(a+b-c)} \\
& \text { Índice de Sorensen }=\frac{2 c}{(a+b)}
\end{aligned}
$$

donde;

$\begin{array}{ll}H^{\prime} & \text { : índice de Shannon. } \\ 1-D & \text { : índice de Simpson. } \\ p_{i} & \text { : proporción de cada especie presente en la muestra. } \\ a & : \text { número de especies presentes en el bosque A. } \\ b & : \text { número de especies presentes en el bosque B. } \\ c & : \text { número de especies presentes en ambos bosques. }\end{array}$

\section{RESULTADOS}

\section{COMPOSICIÓN FLORÍSTICA}

Los bosques costeros presentaron mayor riqueza de especies que los bosques andinos. En los bosques de la costa se registró la presencia de Cryptocarya alba (Molina) Looser, especie de los bosques xerofíticos de la zona central de 
Chile y Aextoxicon punctatum Ruiz et Pavón, especie del tipo forestal Siempreverde. Los bosques costeros y andinos presentaron especies comunes como Gevuina avellana Molina, Persea lingue (Ruiz et Pav.) Nees ex Kopp y $N$. glauca, aunque la localización andina Cerro Castro presentó sólo ésta última especie, siendo por lo tanto, bosque puro de N. glauca.

\section{CARACTERIZACIÓN DE LA ESTRUCTURA}

La estructura de los bosques evaluados se caracterizó por la elevada participación de $N$. glauca, tanto en número de árboles como área basal (Tabla II). La frecuencia relativa del número de árboles de $N$. glauca varió entre 71 y 100 $\%$ en Copiulemu y Cerro Castro localizados en la Costa y Los Andes, respectivamente. De igual manera, la frecuencia relativa de área basal fluctuó entre 88 y $100 \%$, con valores absolutos mínimos de $21,4 \mathrm{~m}^{2} \mathrm{ha}^{-1}$ y máximo de $52,0 \mathrm{~m}^{2}$ ha $^{-1}$ (Tabla II). La segunda especie en importancia fue $G$. avellana, participó entre el 21,0 y 5,6\% de densidad relativa en Copiulemu y Laguna de La Plata, respectivamente. El DMC (diámetro medio cuadrático), que se asocia a la densidad de los rodales, varió entre 15,0 y $18,2 \mathrm{~cm}$ para densidades que fluctuaron entre 1.326 y 2.004 árboles ha- ${ }^{-1}$, respectivamente (Tabla II).

En los bosques evaluados $N$. glauca predominó en todas las clases diamétricas (Fig. 1). En la localización andina de Cerro Castro, $N$. glauca fue la única especie presente. En los otros bosques estudiados, las especies acompañantes $(G$. avellana, $P$. lingue y $C$. alba) tuvieron baja participación en las clases diamétricas menores a $25 \mathrm{~cm}$. La distribución diamétrica de $N$. glauca en Los Queules y Laguna de La Plata (ubicación costera y andina, respectivamente) presentó una clara tendencia del tipo "J invertida". El bosque Cerro Castro tendió a la misma distribución, aunque la clase diamétrica más baja $(5 \mathrm{~cm})$ presentó menos árboles que lo esperado (figura 1), esto significó que la capacidad de regeneración del rodal disminuyera. El rodal presentó alta densidad arbórea (alto grado de ocupación del sitio), presencia de una gruesa capa de hojarasca y bajo grado de luminosidad a nivel del suelo, limitando con ello las condiciones para regeneración de la especie. De igual manera, el bosque Copiulemu tendió a una distribución del tipo señalado, aunque en este caso, no estuvieron presentes las clases diamétricas mayores, debido a corta de árboles adultos que se efectuaron en el pasado, evidenciado en el lugar por la presencia de tocones de mayores diámetros.

En todos los estratos arbóreos $N$. glauca estuvo presente mayoritariamente (Fig. 2). La participación en el estrato dominante varió desde $19 \%$ en Laguna de La Plata, hasta 41 $\%$ en Copiulemu. También en el estrato suprimido N. glauca fue la principal especie, alcanzando la mayor proporción de árboles en Los Queules con el 52 \% y la menor en Cerro Castro con $34 \%$. En el bosque andino de Cerro Castro, donde estuvo presente sólo $N$. glauca, los estratos dominante y codominante concentraron el $67 \%$ de los árboles.

Los bosques costeros de N. glauca presentaron mayor altura que los bosques andinos. La altura media según estrato fue de 11,4 m para árboles suprimidos, $14,7 \mathrm{~m}$ para los codominantes y $20,9 \mathrm{~m}$ para los dominantes (fig. 3). En cambio para los bosques andinos la altura media fue de 8,6 $\mathrm{m}$ para árboles suprimidos, $13,4 \mathrm{~m}$ para los codominantes y $18,0 \mathrm{~m}$ para los dominantes. Al comparar la altura de los árboles dominantes a similar edad, se tiene que los ubicados en los bosques costeros alcanzaron mayor altura que los dominantes de los bosques andinos, es decir, los sitios costeros son de mejor calidad.

TABLA I. Descripción general de los puntos de muestreo según zona.

TABLE I. General description of the points of sampling according to zone.

\begin{tabular}{|c|c|c|c|c|c|}
\hline ZoNA & Bosque & Parcela & $\begin{array}{l}\text { ALTITUD } \\
\text { (m s.n.m.) }\end{array}$ & EXPOSICIÓN & Pendiente $(\%)$ \\
\hline \multirow[t]{6}{*}{ Los Andes } & Laguna de La Plata & 1 & 663 & Noreste & 25 \\
\hline & & 2 & 745 & Noroeste & 35 \\
\hline & & 3 & 646 & Noroeste & 30 \\
\hline & Cerro Castro & 1 & 948 & Noroeste & 20 \\
\hline & & 2 & 962 & Noroeste & 25 \\
\hline & & 3 & 965 & Noroeste & 20 \\
\hline \multirow[t]{6}{*}{ Costa } & Los Queules & 1 & 490 & Noreste & 25 \\
\hline & & 2 & 463 & Noreste & 30 \\
\hline & & 3 & 491 & Noreste & 20 \\
\hline & Copiulemu & 1 & 566 & Noreste & 25 \\
\hline & & 2 & 551 & Noreste & 15 \\
\hline & & 3 & 561 & Noreste & 15 \\
\hline
\end{tabular}


TABLA II. Principales parámetros de los bosques evaluados.

TABLE II. Principal parameters of the evaluated forests.

\begin{tabular}{|c|c|c|c|c|}
\hline EsPECIE & Copiulemu & Los Queules & $\begin{array}{c}\text { Laguna de La } \\
\text { Plata }\end{array}$ & Cerro Castro \\
\hline \multicolumn{5}{|c|}{ Densidad (árboles ha-1) } \\
\hline Gevuina avellana & 279 & 183 & 83 & 0 \\
\hline Persea lingue & 42 & 33 & 33 & 0 \\
\hline Cryptocaria alba & 63 & 83 & 0 & 0 \\
\hline Nothofagus glauca & 942 & 1.283 & 1.375 & 2.004 \\
\hline Aextoxicon punctatum & 0 & 38 & 0 & 0 \\
\hline Total & 1.326 & 1.620 & 1.491 & 2.004 \\
\hline \multicolumn{5}{|c|}{ Densidad relativa (\%) } \\
\hline Gevuina avellana & 21,0 & 11,3 & 5,6 & 0,0 \\
\hline Persea lingue & 3,2 & 2,0 & 2,2 & 0,0 \\
\hline Cryptocaria alba & 4,8 & 5,1 & 0,0 & 0,0 \\
\hline Nothofagus glauca & 71,0 & 79,2 & 92,2 & 100,0 \\
\hline Aextoxicon punctatum & 0,0 & 2,4 & 0,0 & 0,0 \\
\hline Total & 100,0 & 100,0 & 100,0 & 100,0 \\
\hline \multicolumn{5}{|c|}{ Área basal $\left(\mathrm{m}^{2} \mathrm{ha}^{-1}\right)$} \\
\hline Gevuina avellana & 1,6 & 1,7 & 0,8 & 0 \\
\hline Persea lingue & 0,2 & 0,5 & 0,3 & 0 \\
\hline Cryptocaria alba & 0,4 & 0,5 & 0 & 0 \\
\hline Nothofagus glauca & 21,4 & 25,0 & 24,2 & 52,0 \\
\hline Aextoxicon punctatum & 0 & 0,7 & 0 & 0 \\
\hline Total & 23,6 & 28,4 & 25,3 & 52,0 \\
\hline \multicolumn{5}{|c|}{ Área basal relativa (\%) } \\
\hline Gevuina avellana & 6,8 & 6,0 & 3,2 & 0,0 \\
\hline Persea lingue & 0,8 & 1,8 & 1,2 & 0,0 \\
\hline Cryptocaria alba & 1,7 & 1,8 & 0,0 & 0,0 \\
\hline Nothofagus glauca & 90,7 & 88,0 & 95,6 & 100,0 \\
\hline Aextoxicon punctatum & 0,0 & 2,4 & 0,0 & 0,0 \\
\hline Total & 100,0 & 100,0 & 100,0 & 100,0 \\
\hline \multicolumn{5}{|c|}{$\mathrm{DMC}(\mathrm{cm})$} \\
\hline Gevuina avellana & 8,5 & 10,8 & 10,8 & 0 \\
\hline Persea lingue & 7,0 & 14,2 & 10,7 & 0 \\
\hline Cryptocaria alba & 9,0 & 9,1 & 0 & 0 \\
\hline Nothofagus glauca & 17,0 & 15,7 & 15,0 & 18,2 \\
\hline Aextoxicon punctatum & 0 & 15,5 & 0 & 0 \\
\hline Total & 15,0 & 14,9 & 14,7 & 18,2 \\
\hline \multicolumn{5}{|c|}{ Rango de edad (años) } \\
\hline Nothofagus glauca & $14-31$ & $13-27$ & $14-32$ & $17-30$ \\
\hline
\end{tabular}



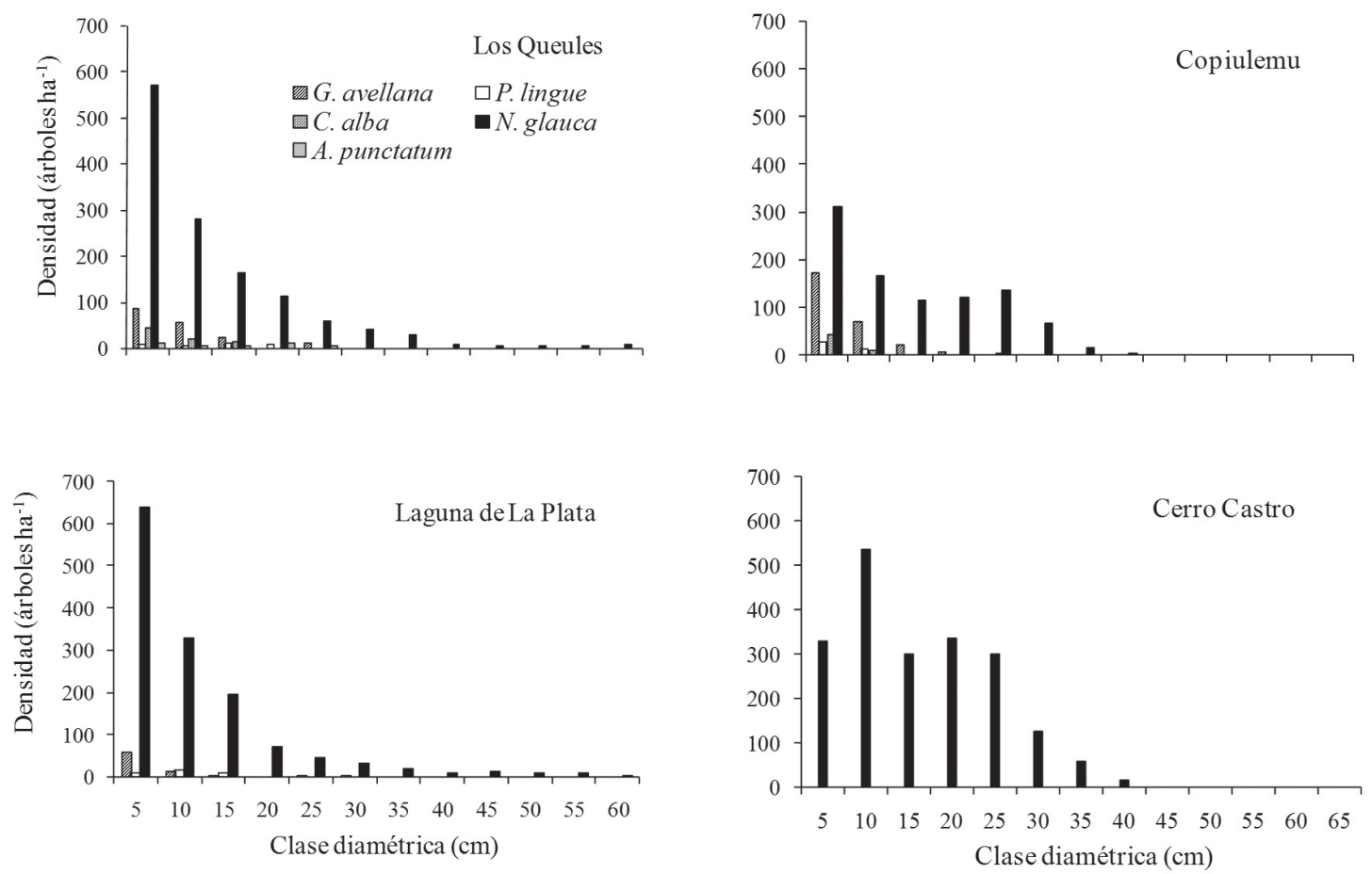

FIGURA 1. Estructura diamétrica según número de árboles y especie en los bosques evaluados.

FIGURE 1. Diameter structure according to the number of trees for each specie in the evaluated forests.
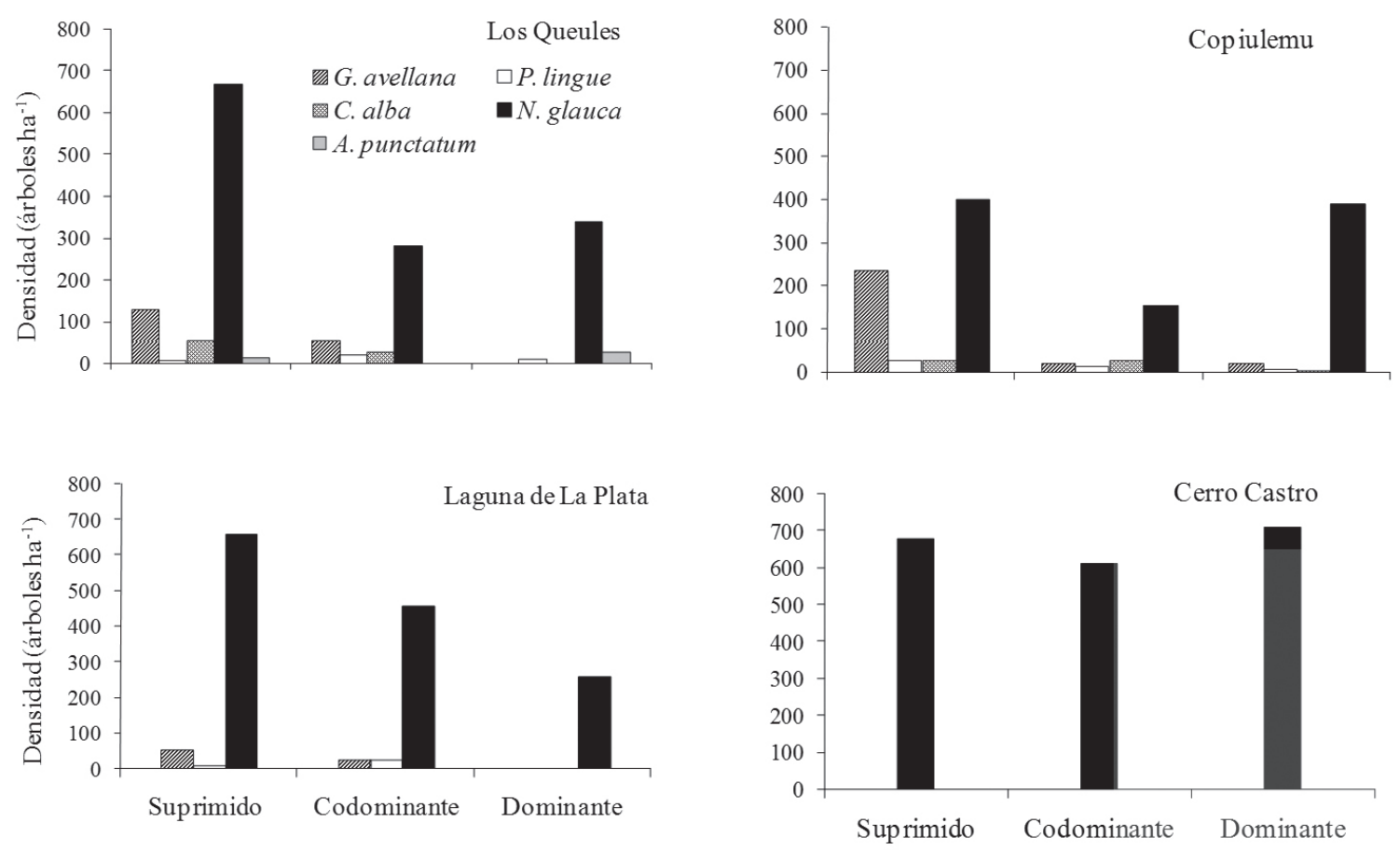

FIGURA 2. Estratificación de copa según especie en cada bosque evaluado.

FIGURE 2. Crown stratification according to species and evaluated forest. 


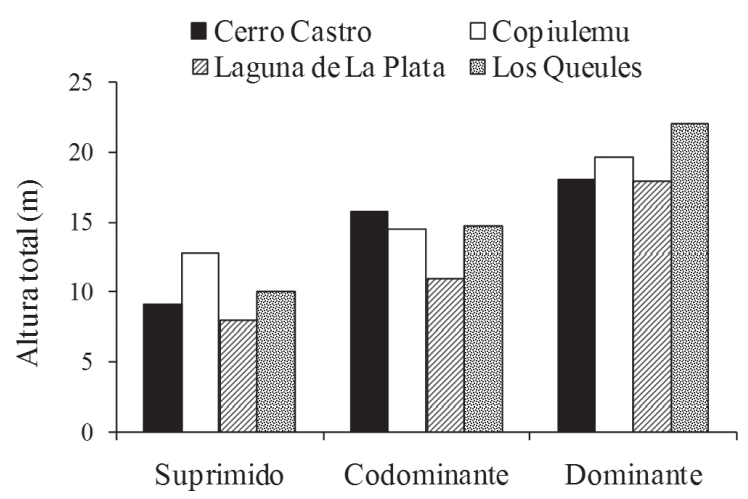

Figura 3. Estratificación de copas de $N$. glauca según altura total (m).

Figure 3. Crown stratification of $N$. glauca according to total height ( $\mathrm{m})$.

\section{Semillación}

Aunque no fue posible cuantificar el total de semillas producidas en cada bosque, fue evidente que los árboles ubicados en los Andes presentaron baja o nula semillación en las dos temporadas de recolección (enero-marzo de los años 2008 y 2009). La cantidad de semilla recolectada en los bosques de los Andes sólo representó el 2,1 \% del total y con mayor proporción de daño (semillas perforadas) que las semillas recolectadas en los bosques de la cordillera de la Costa. En promedio, el 80,9 \% de las semillas recolectadas en la Costa no presentó perforación, en comparación al 54,8 $\%$ de las semillas recolectadas en los Andes.

\section{DIVERSIDAD}

La diversidad dentro de las comunidades (diversidad $\alpha$ ) fue mayor en los bosques costeros. El índice Shannon de diversidad de especies mostró un valor promedio que fue 6,7 veces mayor en la Costa que en los Andes (Tabla III). El bosque andino de Cerro Castro presentó índice 0, indicando con ello que existe sólo una especie. Similar situación ocurrió con el índice Simpson de dominancia de especies con valor promedio 6,5 veces superior en la costa (Tabla III).

La diversidad y dominancia de especies entre comunidades (diversidad $\beta$ ), fue claramente mayor en los bosques costeros. Los índices de diversidad de Jaccard y de Sorensen lo indicaron claramente. Los bosques andinos presentaron índice de Jaccard de 0,3 y Sorensen de 0,5. En cambio, los bosques costeros presentaron valores de $0,8 \mathrm{y}$ 0,9 para los índices Jaccard y Sorensen, respectivamente. Es decir, los índices Jaccard y de Sorensen de los bosques costeros fueron 2,7 y 1,8 veces superiores a los valores de los bosques andinos, respectivamente.

TABla III. Índices de diversidad de especies dentro de comunidades (diversidad $\alpha$ ).

TABLE III. Diversity index of species inside communities (diversity $\alpha$ ).

\begin{tabular}{|c|c|c|c|c|}
\hline \multirow[b]{2}{*}{ ZONA } & \multirow[b]{2}{*}{ Bosque } & \multirow[b]{2}{*}{ Parcela } & \multicolumn{2}{|c|}{ ÍNDICES } \\
\hline & & & SHANNON $\left(H^{\prime}\right)$ & SIMPSON $(1-D)$ \\
\hline \multirow[t]{7}{*}{ Los Andes } & Laguna de La & 1 & 0 & 0 \\
\hline & Plata & 2 & 0 & 0 \\
\hline & & 3 & 0,66 & 0,36 \\
\hline & Cerro Castro & 1 & 0 & 0 \\
\hline & & 2 & 0 & 0 \\
\hline & & 3 & 0 & 0 \\
\hline & Promedio & & 0,11 & 0,06 \\
\hline \multirow[t]{7}{*}{ Costa } & Los Queules & 1 & 0,67 & 0,34 \\
\hline & & 2 & 0,58 & 0,27 \\
\hline & & 3 & 0,93 & 0,46 \\
\hline & Copiulemu & 1 & 0,94 & 0,50 \\
\hline & & 2 & 0,84 & 0,52 \\
\hline & & 3 & 0,50 & 0,24 \\
\hline & Promedio & & 0,74 & 0,39 \\
\hline
\end{tabular}




\section{DISCUSIÓN}

DIFERENCIA ENTRE COMUNIDADES

Se aprecia diferencia entre los bosques de $N$. glauca localizados en sectores costeros y andinos. Los bosques costeros presentan mayor riqueza, dominancia y diversidad de especies que los bosques andinos. Similares resultados de riqueza de especies encontraron Litton \& Santelices (1996) al comparar comunidades de $N$. glauca costeras y andinas en la Región del Maule. Las diferencias de humedad, precipitación y altitud entre los bosques de la costa y los Andes influyen fuertemente en las diferencias florísticas (Litton \& Santelices 1996). De igual manera, a similar edad, las comunidades costeras presentan estrato dominante de mayor altura. Esto señala que los bosques de la costa son de mejor calidad, tal como lo indican estudios desarrollados por Pimstein (1974) que muestran mayor relación carbono/ nitrógeno bajo bosque de $N$. glauca en el sector Coronel de Maule (costa) que en San Fabián de Alico (los Andes). Al respecto, Donoso (2008) señala que, aunque no existen ejemplos debidamente analizados en el bosque chileno, es más probable que una mayor proporción de $N$. obliqua o de $N$. nervosa dentro del tipo de bosque $N$. obliqua $-N$. glauca, en el que predomina $N$. glauca, indique sitio de mejor calidad que aquéllos donde $N$. glauca es puro o la proporción de $N$. obliqua es menor. Es decir, la presencia de otras especies junto a $N$. glauca, en ese caso con otras especies del género Nothofagus, estaría indicando una mejor calidad de sitio. Sin embargo, en este estudio el tamaño de los individuos (área basal y DMC) del bosque puro de $N$. glauca (bosque andino Cerro Castro) es mayor que en los otros sitios estudiados.

En las comunidades de ambos sectores (costero y andino), N. glauca es la especie principal en todos los estratos de copa (dominante, codominante y suprimido), alcanzando el bosque andino Cerro Castro un $100 \%$ de participación (bosque puro). La dominancia de $N$. glauca en todos los estratos se puede explicar por sus efectos alelopáticos, cuya toxicidad máxima se presenta en las hojas caídas en el otoño (Donoso 2008). Adicionalmente, en los bosques de los Andes, la altitud a la cual se localizan limita la presencia de otras especies (Laguna de la Plata varía entre 646-745 m s.n.m. y Cerro Castro varía entre 948-962 m s.n.m.).

\section{ESTRUCTURA DIAMÉTRICA}

La presencia de las especies acompañantes puede ser el resultado de una sucesión secundaria después de un disturbio (tala rasa, incendio, entre otros), iniciándose con el desarrollo de $N$. glauca, debido a su carácter pionero, para posteriormente incorporarse las especies acompañantes mencionadas, tal como Donoso (1993) lo describe para formaciones de $N$. obliqua ubicadas en la cordillera de Parral en la zona central de Chile.

En relación al rodal puro de $N$. glauca (bosque andino Cerro Castro), su distribución diamétrica varía entre $<10$ y $40 \mathrm{~cm}$, densidad de 2004 árboles ha-1, área basal de 52,0 m² $\mathrm{ha}^{-1}$ y DMC de 18,2 cm. Estos valores son similares a los descritos por Pimstein (1974), quien evaluó un bosque puro adulto de $N$. glauca ubicado en Coronel del Maule (sector Costa), de densidad de 2.100 árboles ha $^{-1}$, DAP medio de
$17,5 \mathrm{~cm}$, área basal de $53 \mathrm{~m}^{2} \mathrm{ha}^{-1}$ y altura entre 16 y $17 \mathrm{~m}$. Al respecto Donoso (1993) señala que aparentemente los rodales muestreados por Pimstein (1974) eran en realidad renovales más viejos, en que había operado el autorraleo y se encontraba gran cantidad de individuos suprimidos en los doseles inferiores.

Del análisis del tamaño de los árboles se concluye que, a igual DAP, los árboles adultos de $N$. glauca ubicados en los bosques costeros son entre 3 y $4 \mathrm{~m}$ más altos que los ubicados en los bosques andinos, indicando con ello que son bosques de mayor productividad. En cambio el DAP de los árboles no presenta diferencias entre los bosques costeros y andinos. Esto significa que el ahusamiento de los árboles, expresada como la razón entre altura y DAP de los árboles (Prodan et al. 1997) es mayor en los bosques costeros.

\section{SEMiLlación}

La baja calidad de semillación es otro aspecto a destacar en el estudio realizado. La mejor semillación, en cantidad y calidad, ocurrió en los sitios costeros. Según Donoso (1993) una buena semillación corresponde a 2.000 .000 semillas año ${ }^{1}$ (considera 500 árboles ha ${ }^{-1}$ ), donde el $47 \%$ son viables y las otras semillas están huecas o perforadas por un lepidóptero. En los bosques andinos y a pesar de los esfuerzos realizados durante dos temporadas (años 2008 y 2009), la cantidad de semillas recolectadas desde 12 árboles seleccionados fue baja, sólo un total de 31 semillas, de las cuales el 38 y 71 $\%$ de las semillas estaban perforadas, recolectadas en Cerro Castro y Laguna de La Plata, respectivamente. Huerta et al. (2011) indican que alrededor del 60\% de los frutos presentes en el árbol, en la época de primavera, presentaban daño por lepidóptero en bosques de $N$. glauca ubicados en la Comuna de Empedrado, Región del Maule. El carácter cíclico de la semillación de $N$. glauca también ha sido mencionado en un estudio desarrollado por Burgos et al. (2008), señalando además, que la producción de semillas es mayor en bosque continuo que en fragmentos. También la ciclicidad de las semillas afecta su capacidad germinativa (Santelices et al. 2006). Según Simonetti et al. (2006) el tamaño del bosque (fragmento o continuo) condiciona el nivel de depredación de semillas. Además, mencionan que en el caso de los depredadores predispersión de semillas de N. glauca, como el lepidóptero del género Perzelia, es 1,4 veces menor en los fragmentos que en el bosque continuo y que los depredadores postdispersión, incluyendo roedores, aves y hormigas son, en promedio, 2,2 veces más abundantes en fragmentos. Las semillas que no han sido atacadas por depredadores predispersión tienen mayores probabilidades de ser consumidas por depredadores postdispersión. De hecho, las semillas de $N$. glauca, cuyo embrión no ha sido consumido por larvas de Perzelia sp., tienen tres veces más probabilidades de ser consumida por roedores y aves (Simonetti et al. 2006). Por otra parte, Donoso et al. (1995) señalan que el ataque de insectos se incrementa en años de baja producción de semillas, que es el caso de los sitios de los Andes.

\section{DIVERSIDAD GENÉTICA}

El reducido número de semillas obtenidas en las dos temporadas de recolección (enero-marzo de los años 2008 
y 2009) hizo necesario reconducir el estudio de diversidad genética a un estudio de las posibilidades que ofrecen las proteínas de la semilla de $N$. glauca como marcador molecular para evaluar diversidad. Los resultados están reportados en Martín et al. (2008) y (2010) e indican que las proteínas de reserva pueden ser utilizadas para el estudio de la diversidad genética e hibridación de $N$. glauca, siendo las glutelinas la fracción más adecuada para ello. Según Donoso (1993) los bosques de $N$. glauca han adquirido la particularidad de constituirse en comunidades discretas, con presencia de ecotonos relativamente estrechos entre ellas y con los otros tipos forestales con que se ponen en contacto. De allí que sea necesario continuar investigando la diversidad genética de la especie, especialmente en los bosques costeros que se encuentran sometidos a fuerte presión antrópica, a objeto de identificar las mejores fuentes de germoplasma para fines de conservación y/o restauración.

\section{CONCLUSIONES}

Existe gran diferencia entre los bosques de $N$. glauca costeros y andinos. En la costa los bosques presentan mayor riqueza, dominancia y diversidad de especies que los bosques de los Andes. En los bosques estudiados, $N$. glauca es la especie principal en todos los estratos de copa (dominante, codominante y suprimido), con estructuras diamétricas principalmente del tipo "J invertida". La mejor semillación, en cantidad y calidad, ocurre en los bosques costeros.

\section{AGRADECIMIENTOS}

Este trabajo ha sido financiado por el proyecto DIUC $\mathrm{N}^{\circ}$ 207.142.029-1.0 de la Dirección de Investigación de la Universidad de Concepción (Chile), así como por el proyecto A/9485/07 de la Agencia Española de Cooperación Internacional para el Desarrollo, Ministerio de Asuntos Exteriores (España). Los autores agradecen por su apoyo a la Corporación Nacional Forestal Región del Maule, a la empresa Forestal Celco S.A. y al ingeniero forestal Sr. Mauricio Morales F.

\section{REFERENCIAS}

Benoit, C. 1989. Libro rojo de la flora terrestre de Chile. CONAF. Ministerio de Agricultura. Santiago. 157 pp.

Burgos, A., A. Grez \& R. Bustamante. 2008. Seed production, predispersal seed predation and germination of Nothofagus glauca (Nothofagaceae) in a temperate fragmented forest in Chile. Forest Ecology and Management 255: 1226-1233.

CONAF-CONAMA-BIRF. 1999. Catastro y evaluación de los recursos vegetacionales nativos de Chile. Corporación Nacional Forestal-Comisión Nacional del Medio Ambiente. Santiago, Chile. 89 pp.

CONAMA. 2012. Lista de especies nativas según estado de conservación. Consultado 18 diciembre 2012. Disponible en http://www.mma.gob.cl/clasificacionespecies.

Daniel, T., J. Helms \& F. Baker. 1982. Principios de Silvicultura. Primera Edición en Español. Mc Graw-Hill. México. $492 \mathrm{pp}$.

Del Fierro, P. \& L. Pancel. 1998. Experiencia silvicultural del Bosque Nativo de Chile. GTZ-CONAF. 420 pp.

Del Río, M., F. Montes, I. Cañellas \& G. Montero. 2003. Índices de diversidad estructural en masas forestales. Investigación Agraria: Sistemas y Recursos Forestales 12(1): 159-176.

Donoso, C. 1993. Bosques templados de Chile y Argentina. Variación, estructura y dinámica. Editorial Universitaria. Segunda Edición. Santiago, Chile. 484 pp.

Donoso, C. 2008. Ecología forestal. El bosque y su medio ambiente. Sexta edición. Editorial Universitaria. Santiago, Chile. $369 \mathrm{pp}$.

Donoso, C., B. Escobar \& M. González. 1995. Técnicas de viveros y plantaciones para hualo (Nothofagus glauca). Documento Técnico No 86 , Chile Forestal, Corporación Nacional Forestal, Chile. 8 pp.

Hechenleitner, P., M. Gardner, P. Thomas, C. Echeverría, B. Escobar, P. Brownless \& C. Martínez. 2005. Plantas amenazadas del Centro-Sur de Chile. Distribución, conservación y propagación. Primera edición. Universidad Austral de Chile y Real Jardín Botánico de Edimburgo. Valdivia. $188 \mathrm{pp}$.

Huerta, A., W. Navarrete, J. Araya \& F. Muñoz. 2011. Composición y clasificación de daño de insectos en Nothofagus glauca (Fagaceae), Región del Maule, Chile. Revista Colombiana de Entomología 37(1): 56-61.

IUCN. 2012. IUCN Red List of Threatened Species. Version 2012.2. <www.iucnredlist.org>. Downloaded on 23 December 2012.

Le-Quesne, C. \& L. Sandoval. 2001. Extensión del límite sur para Nothofagus glauca (Phil.) Krasser. Gayana Botánica 58(2): 139-142.

Litton, C.\& R. SAntelices. 1996. Comparación de las comunidades vegetales en bosques de Nothofagus glauca (Phil.) Krasser en la Séptima Región de Chile. Bosque 17(2): 77-86.

López, E. 1994. Caracterización bioclimática de la Estación Meteorológica de la Universidad Católica del Maule. Taller de Habilitación Profesional, Facultad de Ciencias Agrarias y Forestales, Universidad Católica del Maule, Talca, Chile 50 pp.

Magurran, AE. 1989. Diversidad ecológica y su medición. Ediciones Vedrá, Barcelona, España. 200 pp.

Martín, M.A., S. Muñoz, F. Muñoz, M. Uribe, J.B. Álvarez, M.A. Herrera \& L.M. Martín. 2008. Recursos genéticos de Nothofagus spp. IV Congreso de Mejora Genética de Plantas, Sociedad Española de Genética, Córdoba, España. 14-16 octubre 2008.

Martín, M.A., S. Muñoz, F. Muñoz, M. Uribe, J. Molina, M.A. Herrera, L.M. Martín \& J.B. Álvarez. 2010. Primeros resultados en el desarrollo de un marcador genético basado en las proteínas de reserva en dos especies del género Nothofagus. Bosque 31(3): 252-257.

Novoa, R. \& S. Villaseca. 1989. Mapa Agroclimático de Chile. Instituto de Investigaciones Agropecuarias (INIA), Ministerio de Agricultura, Santiago, Chile. 221 pp. 
Pauchard, A., M. Aguayo \& P. Alaback. 2006. Cuantificando la fragmentación del paisaje: las métricas y sus significados ecológicos. In A. Grez, J. Simonetti, R. Bustamante (eds). Biodiversidad en ambientes fragmentados de Chile: Patrones y procesos a diferentes escalas. Editorial Universitaria S.A., Santiago, Chile. 41-67 pp.

Pimstein, R. 1974. Contribución al estudio de ecosistemas en comunidades de roble maulino (Nothofagus glauca). Tesis Ingeniería Forestal. Facultad Ciencias Forestales, Universidad de Chile. Santiago, Chile. 96 pp.

Prodan, M., R. Peters, F. Cox \& P. Real. 1997. Mensura Forestal. Serie Investigación y Educación en Desarrollo Sostenible. IICA-GTZ. San José, Costa Rica. 561 pp.

Santelices, R., C. Donoso \& A. Cabello. 2006. Nothofagus glauca (Phil.) Krasser, Hualo, Roble maulino, Roble colorado (Maule). Familia: Fagaceae. In Donoso C, (Ed). Las Especies Arbóreas de los Bosques Templados de Chile y
Argentina: Autoecología. Marisa Cuneo Ediciones. 433$442 \mathrm{pp}$.

Serra, M., R. Fajardo \& A. Cabello. 1986. Ficha técnica de especies amenazadas: Nothofagus glauca (Phil.) Krasser, Hualo (Fagaceae) especie vulnerable. Programa de Protección y Recuperación de la Flora de Chile, CONAF/UCH, Santiago.

Simonetti, J., A. Grez \& R. Bustamante. 2006. Interacciones y procesos en el bosque maulino. In: A. Grez, J. Simonetti, R. Bustamante (eds.). Biodiversidad en ambientes fragmentados de Chile: Patrones y procesos a diferentes escalas. Editorial Universitaria S.A., Santiago, Chile. 99-114 pp.

UrzúA, A. 1975. Cambio de estructura en el bosque de Nothofagus glauca (Phil.) Krasser. Tesis Ingeniería Forestal. Facultad Ciencias Forestales, Universidad de Chile, Santiago, Chile. 38 pp.

Recibido: 18.07 .12

Aceptado: 11.02.13 